\title{
A note on quasi-uniform continuity
}

\section{Panayotis Th. Lambrinos}

It is shown that:

(i) a continuous function from a compact quasi-uniform space into a quasi-uniform space is not necessarily quasiuniformly continuous;

(ii) if the range of the function is a uniform space, the function will be necessarily quasi-uniformly continuous.

(This contradicts an example in the literature and generalizes a classical result.) Finally, a generalization of (ii) is given by means of a suitable boundedness notion.

A quasi-uniform space is defined to be a pair $(X, U)$, where $X$ is a non-empty set and $U$ is a filter on $X \times X$ which satisfies:

(i) each $U \in U$ defines a reflexive relation on $X$,

(ii) to each $U \in U$ there corresponds $W \in U$ such that $W \circ W \subseteq U$.

The pair $(X, U)$ becomes a uniform space when:

(iii) $U^{-1} \in U$ whenever $U \in U$.

Examples of quasi-uniform spaces which are not uniform are easily found using the fact that every topological space is quasi-uniformizable [5, Theorem 1.19]. On the other hand, each quasi-uniform space $(X, U)$ gives rise to a topology on $X$, the neighbourhood system for each $x \in X$ being given by the family

$$
U(x) \equiv\{y \in X:(x, y) \in U\}, \quad U \in U \text {. }
$$


It is well-known [3, p. 198] that each continuous function from a compact uniform space into a uniform space is uniformly continuous and that there exist compact quasi-uniform spaces the quasi-uniformity of which is not a uniformity [2, p. 399 , Example]. Here we give

THEOREM 1. Every continuous function from a compact quasi-uniform space into a uniform space is quasi-uniformly continuous.

REMARK 1. This result contradicts an example, in [5, p. 55], given without proof, of a continuous function from a compact quasi-uniform space into a uniform space which is not quasi-uniformly continuous.

Proof. See Remark 3.

COUNTEREXAMPLE. The above result is best possible in the sense that a continuous function from a compact quasi-uniform space (even a compact uniform space) into a quasi-uniform space is not necessarily quasiuniformly continuous. For suppose that $X$ is a uniform (with uniformity U) non-discrete compact $T_{0}$ space and that $P$ is the Pervin quasiuniformity for $X[5, \mathrm{p} .15]$. The identity map from $(X, U)$ into $(X, P)$ is continuous but it is not quasi-uniformly continuous since, according to $[2$, p. 398, Theorem 5], P\$U.

In order to state and prove Theorem 2 below, we have need of the following, which are proved in [4].

DEFINITION. A subset $A$ of a space $(X, r)$ is said to be r-bounded if from each open cover of $X$ one can select a finite subcover of $A$; equivalently: if every filter in $A$ has at least one adherent point in the space $(X, r)$.

REMARK 2. From the above and Exercise $1 \mathrm{~b}$ in $[1, \mathrm{p} .109]$ it follows that there exist spaces such that an $r$-bounded subset is not necessarily compact or relatively compact (that is, subset of a compact set). However each (relatively) compact subset is r-bounded.

PROPOSITION. Although a space $(X, r)$ is compact if and only if $X$ is r-bounded, a proper subset A may be $r$-bounded without the relative space $\left(A, r_{A}\right)$ being compact, that $i s$, without $A$ being $r_{A}$-bounded.

(See Remark 2.)

THEOREM 2. Let $f:(X, R) \rightarrow(Y, U)$ be a continuous function from 
the quasi-uniform space $(X, R)$ into the uniform space $(Y, U)$ and let $A$ be an r-bounded subset of $X$. Then the restriction $f / A:\left(A, R_{A}\right) \rightarrow(Y, U)$ of the function $f$ to $A$ is quasi-uniformly continuous (with respect to the relative quasi-uniformity $R_{A}$, induced by $R$ on $A$ ).

Proof. To show that $f / A$ is quesi-uniformly continuous we must show that for every $U \in U$, there exists $V \in R$ satisfying

$$
\{(f(x), f(y)):(x, y) \in V \cap(A \times A)\} \subseteq U \text {. }
$$

There exists symmetric $W \in U$ such that $W \circ W \subseteq U$. Let $x \in X$. Since $f$ is continuous, corresponding to the neighbourhood $W(f(x))$ of $f(x)$, there exists $V_{x} \in R$ such that

$$
f\left(V_{x}(x)\right) \subseteq W(f(x))
$$

Choose $T_{x} \in R$ such that $T_{x} \circ T_{x} \subseteq V_{x}$. The union of the interiors of the $T_{x}(x)$ covers $X$ and so the r-boundedness of $A$ implies that there exists a finite set of points $\left\{x_{1}, \ldots, x_{n}\right\}$ in $X$ satisfying

$\bigcup_{k=1}^{n} T_{x_{k}}\left(x_{k}\right) \supseteq A$. . Define $V=\prod_{k=1}^{n} T_{x_{k}} \in R$. We will show that $V$ satisfies (1).

Suppose that $(a, b) \in V \cap(A \times A)$. Then there exists $k \in\{1, \ldots, n\}, k=k(a)$, such that $a \in T_{x_{k}}\left(x_{k}\right)$. Thus $\left(x_{k}, a\right) \in T_{x_{k}}$. But also $(a, b) \in T_{x_{k}}$, hence $\left(x_{k}, b\right) \in T_{x_{k}} \circ T_{x_{k}} \subseteq V_{x_{k}}$ and so $b \in V_{x_{k}}\left(x_{k}\right)$. Accordingly $a, b \in V_{x_{k}}\left(x_{k}\right)$. Using (2) we have

$$
f(a), f(b) \in f\left(v_{x_{k}}\left(x_{k}\right)\right) \subseteq W\left(f\left(x_{k}\right)\right),
$$

hence $\left(f(a), f\left(x_{k}\right)\right) \in W^{-1}=W$ and $\left(f\left(x_{k}\right), f(b)\right) \in W$. Therefore $(f(a), f(b)) \in W \circ W \subseteq U$ as required.

REMARK 3 . If $X$ is $r$-bounded, by the Proposition we get Theorem 1 as a special case of Theorem 2 above. 


\section{References}

[1] N. Bourbaki, 'Eléments de mathématique, Livre III, Topologie générale, $2 e$ édition, Chapitres I et II (Hermann, Paris, 1951).

[2] P. Fletcher, "On totally bounded quasi-uniform spaces", Arch. Math. 21 (1970), 396-401.

[3] John L. Kelley, General topology (Van Nostrand, Toronto, New York, London, 1955).

[4] Panayotis Lambrinos, "A topological notion of boundedness", submitted.

[5] M.G. Murdeshwar and S.A. Naimpally; Quasi-uniform topological spaces (Noordhoff, Series A: Preprints of Research Papers, No. 4, Vol. 2, 1966).

Department of Mathematics, Aristotle University of Thessaloniki,

Thessaloniki,

Greece. 